\title{
Resonant X-ray diffraction of self-assembled epitaxial systems: From direct to complementary chemical information
}

\author{
L.N. Coelho ${ }^{1}$, B. Diaz ${ }^{2}$, R. Magalhães-Paniago ${ }^{3}$, and A. Malachias ${ }^{2,3, a}$ \\ 1 Instituto de Física, Universidade de Brasília, Brasília DF, CEP 70910-900, Brazil \\ 2 Laboratório Nacional de Luz Síncrotron, C.P. 6192, Campinas, SP, CEP 13085-903, Brazil \\ 3 Departamento de Física, Universidade Federal de Minas Gerais, CP 702, Belo Horizonte, \\ MG, Brazil
}

Received 4 December 2011 / Received in final form 23 March 2012

Published online 15 June 2012

\begin{abstract}
In this work we depict schematically the use of resonant (anomalous) X-ray diffraction as a tool to directly probe strain and composition of self-assembled semiconductor islands. By employing a direct analysis at the $\mathrm{Eu} \mathrm{L}_{3}$ edge its composition gradient is quantified for EuTe:SnTe capped islands. Projection maps are proposed to visualize the results, providing an alternative capability to infer quantum dot properties. A more complex methodology is applied to the study of InP:GaAs islands, in which complementary anomalous measurements are performed. For this system the number of samples analyzed allows us to extract the activation energy for Ga adatoms diffusion from the substrate to the islands.
\end{abstract}

\section{Introduction}

Resonant X-ray scattering has been explored in crystallography since the early experiments in X-ray tubes [1]. With the advent of synchrotron radiation the selection of one or more specific X-ray energies for performing scattering/diffraction experiments became easier due to the availability of such continuous spectrum sources [2]. X-ray diffraction is a non-destructive technique and can provide statistical information over large areas of the studied materials. Its use to investigate self-assembled quantum dots spans from the first grazing-incidence diffraction experiments [3] to the recent achievements in coherent diffraction imaging [4]. The description of nanostructures by $\mathrm{X}$-ray methods has, therefore, remarkably evolved towards quantitative tomographic results. In particular, anomalous (resonant) scattering has played a crucial role in this field, depicting composition gradients that are mandatory to retrieve the complete scenario of nucleation and growth processes which take place during epitaxial island formation [5].

\footnotetext{
a e-mail: angelomalachias@yahoo.com
} 
Hetero-epitaxial self-assembled islands and quantum dots (QDs) have provided to the scientific community interesting systems not only for their quantum confinement capabilities but also for possible fine tuning of such properties in the design of devices [6,7]. This tuning, however, is not simple, since several aspects influence the physical properties of QDs, such as chemical composition, island size, strain, capping, among others. The pseudomorphic growth of QDs on a substrate with a different lattice parameter leads to the formation of elastically strained three-dimensional structures [8] in which strain relaxation can take place in the form of lattice parameter gradients [3] or by the creation of dislocations. The latter process renders the dots optically inactive, while elastic relaxation by strain and composition gradients may lead to optically active QDs with very different properties with respect to bulk materials [9]. Complete structural and chemical description of the QDs is, therefore, crucial to determine which growth parameters yield the desired properties and, by understanding the final scenario, infer the overall electronic behavior of such nanoobjects.

Intensive work has been done to determine the dependence of optical and electronic properties on concentration for binary - such as Ge:Si(001) [6] - and ternary III-V systems - e.g., InAs/InAsP [7] and InGaAs/GaAs(001) [7]. However, little is known about ternary IV-VI magnetic QDs as well as for quaternary III-V systems. Resonant X-ray Diffraction (RXD) [10-14] in grazing incidence geometry (GID) has indicated, along with Electron Energy Loss Spectroscopy (EELS) [15], that none of these QDs are free from substrate atoms. A probe that determines simultaneously the concentration of each element inside the QDs as well as the strain they are submitted is thus necessary to better understand the influence of growth parameters on the final characteristics of the islands.

In this review we show how the combination of iso-strain scattering and anomalous diffraction methods can be employed to retrieve lattice parameter and composition profiles for EuTe:SnTe and InP:GaAs islands. A projected visualization is proposed to interpret the Eu concentration distribution in the buried islands of the first system, while the activation energy for Ga adatom incorporation is obtained for III-V islands.

The paper is ordered as follows: we first depict strain and composition of EuTe:SnTe islands, by directly accessing the $\mathrm{Eu} \mathrm{L}_{3}$ absorption edge. Secondly, InP:GaAs islands are studied by means of complementary anomalous diffraction at the $\mathrm{Ga}$ and As edges (In and $\mathrm{P}$ edges are out of the energy range accessible by our experimental setup).

\section{Experimental}

Grazing-incidence X-ray diffraction measurements were performed at beamlines XRD1 and XRD2 of the Brazilian Synchrotron Light Laboratory (LNLS), in Campinas. Both facilities are equipped with similar X-ray optics, including a cylindrically bent Rh-coated mirror, used to suppress high energy harmonics and focus the beam vertically. The diffractometers used have independent circles allowing to tune the incident angle for measurements at GID geometry. At the XRD2 beamline a $4+2$ circle diffractometer was used while at XRD1 the measurements were performed in a 2-circle diffractometer with an independent $\alpha_{\mathrm{i}}$ arc. The scattered signal was integrated over the exit angle $\alpha_{\mathrm{f}}$ ranging from $0^{\circ}$ to $1.5^{\circ}$ in both beamline setups [3]. Although high momentum transfer measurements allow for more pronounced anomalous effect - as explored in ref. [12] - we have restricted our analysis to the most intense reflection at low momentum transfer [i.e. (220)] since weak bending magnet sources at a second generation synchrotron were used. Whenever higher photon flux or larger acquisition times can be used for this kind of resonant experiment is it useful 
to access high-momentum reflections such as (440), (800), etc, allowing for enhanced chemical contrast.

EuTe islands were grown at $200^{\circ} \mathrm{C}$ by molecular beam epitaxy (MBE) on a $\mathrm{BaF}_{2}(111)$ freshly cleaved substrate of $15 \times 15 \mathrm{~mm}$. To clean the substrate surface from adsorbed water and impurities, it was first heated to $150{ }^{\circ} \mathrm{C}$ during $30 \mathrm{~min}$ in the MBE preparation chamber, and then to $400^{\circ} \mathrm{C}$ during $15 \mathrm{~min}$ in the growth chamber. The substrate was then covered with a $2 \mu \mathrm{m}$-thick SnTe buffer obtained using a compound SnTe source. During the growth of the EuTe dots, a Eu source and two Te sources were used. EuTe growth details are discussed in ref. [16]. Finally, the islands were capped by 20 monolayers (ML) of SnTe to protect them from oxidation.

Four samples of uncapped InP islands were grown by Chemical Beam Epitaxy on a clean $\mathrm{GaAs}(001)$ substrate after oxide layer removal at $600{ }^{\circ} \mathrm{C}$ for 15 minutes. A GaAs buffer layer was grown at $550^{\circ} \mathrm{C}$ for $30 \mathrm{~min}$ in arsine $\left(\mathrm{AsH}_{3}\right)$ atmosphere using triethylgallium (TEGa) as precursor for Ga atoms. The temperature was then lowered to the growth temperature $(\mathrm{Tg})$ of the QDs: $\mathrm{T}_{\mathrm{G}}=470{ }^{\circ} \mathrm{C}$ (sample $\mathrm{A}$ ), $\mathrm{T}_{\mathrm{G}}=500{ }^{\circ} \mathrm{C}$ (sample B) and $\mathrm{T}_{\mathrm{G}}=530^{\circ} \mathrm{C}$ (sample $\mathrm{C}$ ). The QDs were grown on thermally cracked phosphine (PH3) atmosphere and Trimethyl Indium (TMIn) was used as precursor for In atoms. Eight equivalent monolayers of $\mathrm{InP}$ were grown at a growth rate of $0.2 \mathrm{EQ}-\mathrm{ML} / \mathrm{s}$. During the entire growth process, the RHEED profile of the sample was followed. The In shutter was closed as soon as the winged pattern of the QDs appears on the RHEED profile. The temperature was then lowered to room temperature in $\mathrm{PH}_{3}$ atmosphere. A fourth sample (sample D) was grown at $\mathrm{T}_{\mathrm{G}}=500{ }^{\circ} \mathrm{C}$ but with the In-flux reduced to $50 \%$ its original value, corresponding to a growth rate of $0.1 \mathrm{EQ}-\mathrm{ML} / \mathrm{s}$. The deposition time for this sample was longer so that the same amount of material was deposited, i.e., 8 equivalent monolayers [17].

\section{Results and discussion}

\subsection{EuTe islands on SnTe}

The europium chalcogenides $\operatorname{EuX}(\mathrm{X}=\mathrm{O}, \mathrm{S}, \mathrm{Se}, \mathrm{Te})$ are magnetic semiconductors, considered model Heisenberg magnets, with strong and localized magnetic moments, that can find applications in prototype spintronic devices. Studied since the sixties, they have recently attracted renewed attention due to the availability of high quality MBE grown samples. Within this family, EuTe is an antiferromagnetic (AFM) material with AFM type II ordering. New adsorption and photoluminescence lines were discovered in EuTe films, as well as magnetic correlations in EuTe/PbTe superlattices, all grown by MBE [18-20].

Heterostructures and ternaries of $\operatorname{EuX}(\mathrm{X}=\mathrm{O}, \mathrm{S}, \mathrm{Se}, \mathrm{Te})$ and IV-VI semiconductors can be easily obtained since these families share the same $\mathrm{NaCl}$ structure and have similar lattice parameters. Ordered stacks of PbSe non magnetic quantum dots with $\mathrm{PbEuTe}$ spacers have been grown, controlling dots arrangements with the spacer thickness [21].

AFM images of the sample show almost only the cap layer with narrow trenches. In other samples, partially covered with only 10 ML SnTe cap layer, EuTe lens-shaped QDs are observed, with a $100 \AA$ radius and $20 \AA$ height (Fig. 1) [16]. In order to quantify the Sn interdiffusion in the EuTe QDs, we used X-ray anomalous diffraction. The measurements were performed at beamline XRD2 at LNLS. The Eu absorption edge with higher contrast reachable in our beamline was $\mathrm{Eu} \mathrm{L}_{3}$, at $6970 \mathrm{eV}$. Near the Eu $\mathrm{L}_{3}$ edge, the scattering factor of the other elements present in the sample, $\mathrm{Sn}$ and Te, remain almost constant [Fig. 2(a)].

To find the values of $\mathrm{f}^{\prime}$ and $\mathrm{f}^{\prime \prime}$ we first measured the fluorescence signal from an EuTe thin film in an interval of $160 \mathrm{eV}$ centered at the tabulated $\mathrm{Eu} \mathrm{L}_{3}$ absorption 


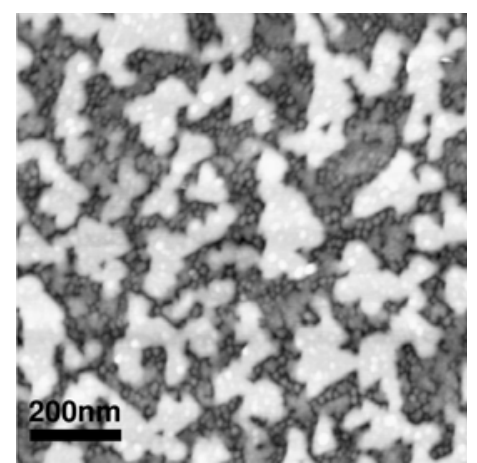

Fig. 1. Atomic force microscopy image of $8 \mathrm{ML}$ EuTe QDs with $10 \mathrm{ML}$ SnTe cap layer. See ref. [16] for more details.
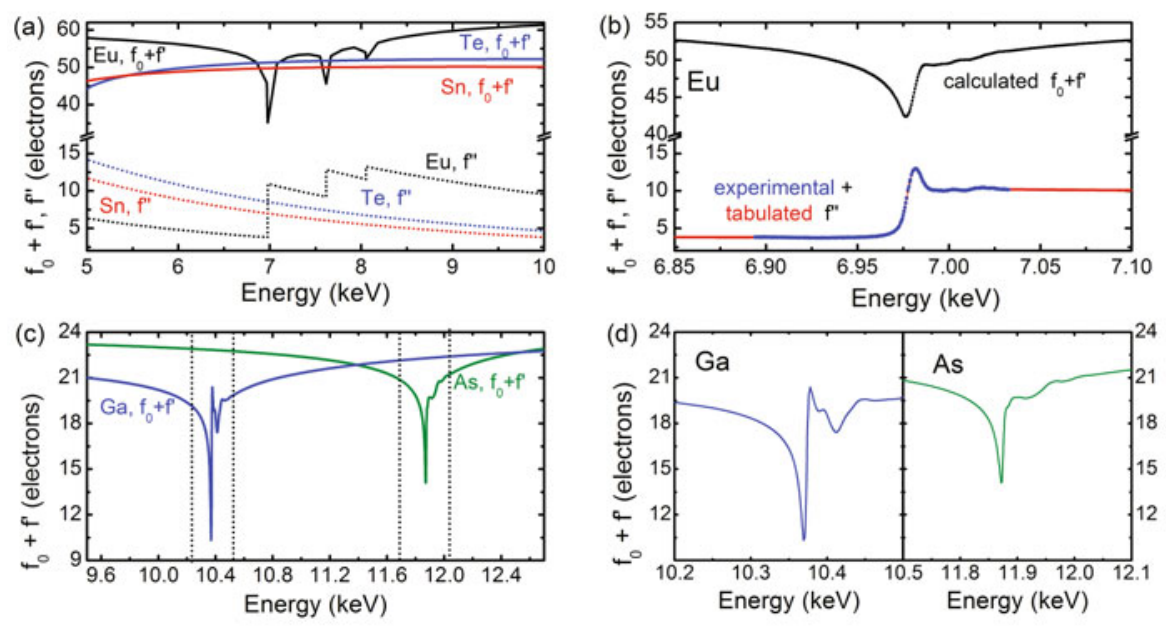

Fig. 2. Atomic scattering factors for all atomic species studied in this work as a function of photon energy. The values of $f_{0}$ were corrected according to the $q$ value for the $(220)$ reflection in EuTe and InP systems, as described in the text. (a) Theoretical real $\left(f_{0}+f^{\prime}\right)$ and imaginary $\left(f^{\prime \prime}\right)$ parts of the atomic scattering factor for Eu, Te and Sn. (b) Calculation of $f_{0}+f^{\prime}$ from $f^{\prime \prime}$ measurements for Eu, following ref. [1]. (c) Measured $f_{0}+f^{\prime}$ for Ga and As edges. (d) Detail of retrieved real part of atomic scattering factors for As and Ga.

edge. This measurement was normalized to fit into the tabulated $\mathrm{f}^{\prime \prime}$ values for $\mathrm{Eu}$ in a much wider energy range. f' values were then calculated using the Kramers-Kronig dispersion relation (Fig. 2(b)), following ref. [1].

The variation in the atomic scattering factor near the Eu absorption edge results in different scattered intensities of the diffraction patterns measured at different energies. This can be readily observed in the longitudinal scans along the $h k l(2-20)$ SnTe reflection, measured at the absorption edge and $150 \mathrm{eV}$ below $\left(\mathrm{E}_{1}=6820 \mathrm{eV}\right.$, and $\mathrm{E}_{2}=6971 \mathrm{eV}$ ) [Fig. 3(a)]. The narrow peak at $\mathrm{h}=2$ corresponds to the $\mathrm{SnTe}$ buffer layer. A broad shoulder appears to the left of the SnTe peak, resulting from the scattering of the EuTe quantum dots $\left(\mathrm{a}_{\mathrm{SnTe}}=6.327 \AA\right.$ and $\mathrm{a} \mathrm{EuTe}=6.598 \AA$ for bulk). As expected, the two scans in Fig. 3 differ mainly in the EuTe QDs shoulder region [10].

The Eu concentration in the QDs can be extracted as a function of local lattice parameter from the diffraction patterns measured at two different energies. Both 


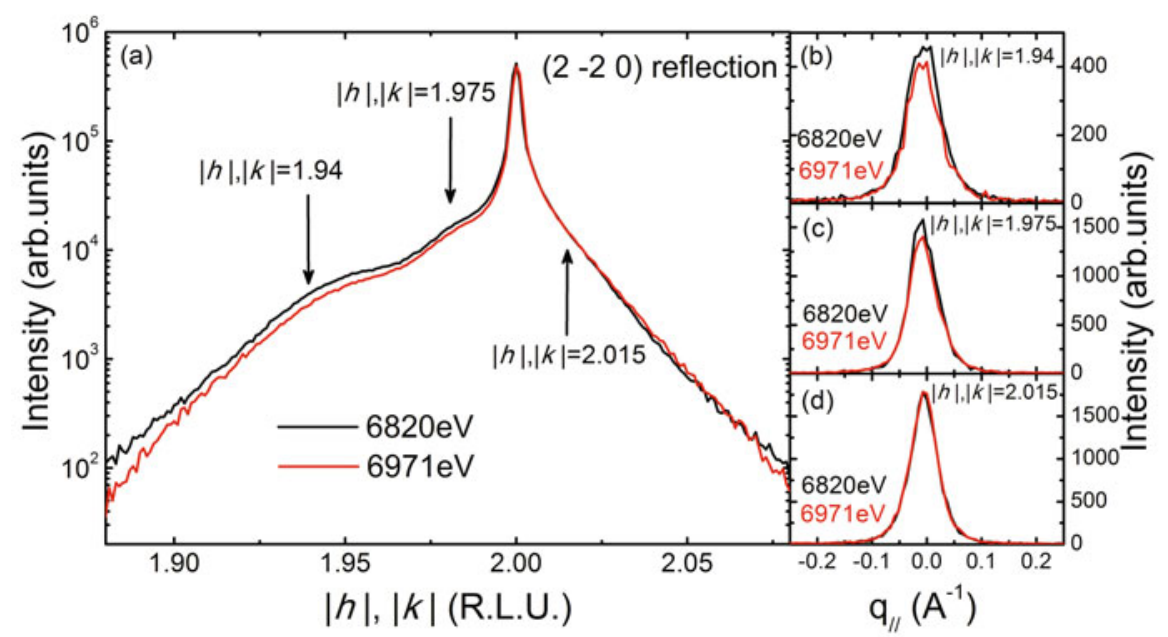

Fig. 3. (a) Grazing incidence longitudinal diffraction scans measured at two different energies: $6820 \mathrm{eV}$ ( $151 \mathrm{eV}$ below the absorption edge) and $6971 \mathrm{eV}$ (at the absorption edge). Angular scans measured below the absorption edge and at the absorption edge, at selected $h$ positions: (b) $h=1.94$, (c) $h=1.975$ and (d) $h=2.015$.

EuTe and SnTe have fcc structures. Hence, for the hkl (2-20) reflection, the structure factor can be calculated as $|\mathrm{F}|^{2}=16\left(\mathrm{f}_{\mathrm{Eu}}+\mathrm{f}_{\mathrm{Te}}\right)^{2}$, where $\mathrm{f}_{\mathrm{Eu}}$ and $\mathrm{f}_{\mathrm{Te}}$ are the atomic scattering factors for $\mathrm{Eu}$ and $\mathrm{Te}$, respectively. If there is interdiffusion of $\mathrm{Sn}$ from the SnTe buffer layer within the EuTe dots, the diffracted intensity for the QDs will be the square of the $\mathrm{Eu}$ concentration $\left(C_{\mathrm{Eu}}\right)$ weighted sum of the atomic factors of $\mathrm{Eu}$ and Sn:

$$
I \propto\left|\left[C_{E u} f_{E u}+\left(1-C_{E u}\right) f_{S n}\right]+f_{T e}\right|^{2} .
$$

Since the atomic scattering factors depend on the energy of the X-rays, the measured intensity will vary with energy as shown in Fig. 2(b). The term $C_{\mathrm{Eu}}$ from Eq. (1) can be retrieved by calculating the intensity ratio of measurements with different energies:

$$
\frac{I_{1}}{I_{2}}=\frac{\left|\left[C_{E u} f_{E u_{1}}+\left(1-C_{E u}\right) f_{S n}\right]+f_{T e}\right|^{2}}{\left|\left[C_{E u} f_{E u_{2}}+\left(1-C_{E u}\right) f_{S n}\right]+f_{T e}\right|^{2}},
$$

where sub-indexes 1 and 2 refer to measurements at different energies $E_{1}$ and $E_{2}$. The resulting equation for $C_{\mathrm{Eu}}$ can be written as:

$$
C_{E u}=\frac{\left[\sqrt{I_{2}}-\sqrt{I_{1}}\right]\left[f_{S n}+f_{T e}\right]}{\sqrt{I_{1}}\left[f_{E u_{2}}-f_{S n}\right]-\sqrt{I_{2}}\left[f_{E u_{1}}-f_{S n}\right]} .
$$

The equation above can be evaluated providing the Eu concentration $C_{\mathrm{Eu}}$ at any two points of the longitudinal scans. In Fig. 3(b, c), we show angular scans measured at $h=1.94$ and 1.975 [marked in Fig. 3(a) with arrows], at two energies. Angular scans at $h=2.015$ (where no Eu contrast effect is expected) were also compared to check if any background or intensity normalization was necessary, but they matched perfectly as shown in Fig. 3(d). Using the integrated intensities of the angular scans at $h=1.94$ and 1.975 one can also calculate the Eu concentration, yielding values of 0.6 and 0.3 , for $h=1.94$ and $h=1.975$, respectively. The peak widths of the angular scans are the same for the two energies. These widths decrease for higher strain (or low $\mathrm{H}$ ) regions, consequently showing that islands are more strained at 

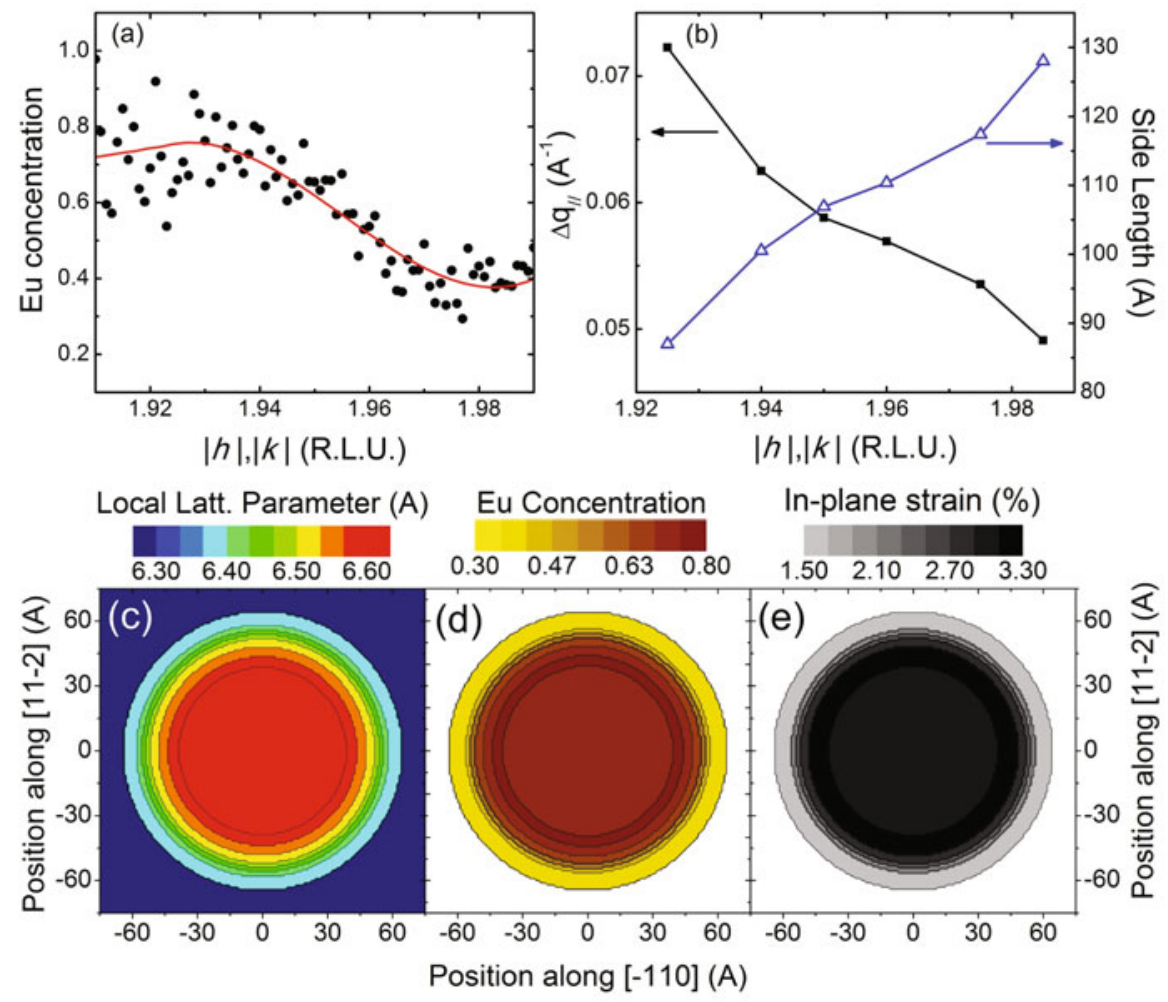

Fig. 4. (a) Eu concentration profile as a function of reciprocal space position (and consequently in-plane lattice parameter) for the EuTe islands studied in this work. The solid line is a polynomial fit to the experimental data. (b) Angular scan widths (squares) and lateral size (open triangles) of iso-lattice parameter regions obtained at the vicinity of the SnTe (220) reflection. Projection reconstructions of local lattice parameter (c), Eu concentration (d) and in-plane strain (e) extracted from the datasets of (a) and (b) are shown.

the island base. The island diameters derived from these widths range from $80 \AA$ to $130 \AA$.

Figure 4(a) shows the Eu concentration as a function of $(\mathrm{H}, \mathrm{K})$ obtained from the longitudinal scans, in good agreement with the isolated results from the angular scans. The solid line is a polynomial fit to the data. The fit reaches a maximum at $\mathrm{H} \sim 1.925$, yielding a lattice parameter of $6.57 \AA$, $0.4 \%$ away from the bulk value of $6.598 \AA$. Even in this region the Sn interdiffusion is significant, about $25 \%$ of the $\mathrm{Eu}$ atoms substituted by Sn according to the anomalous diffraction result. This is in apparent contradiction with the narrow miscibility range of the $\mathrm{Sn}_{1-x} \mathrm{Eu}_{x} \mathrm{Te}$ ternary. For bulk unstrained crystals of $\mathrm{Sn}_{1-x} \mathrm{Eu}_{x} \mathrm{Te}$ with $\mathrm{x} \sim 0.02$, EuTe clusters are already observed. Miscibility in $\mathrm{Sn}_{1-x} \mathrm{Eu}_{x} \mathrm{Te}$ is hindered due to the $4.3 \%$ lattice mismatch between $\mathrm{EuTe}$ and $\mathrm{SnTe}$, and the $20 \%$ difference in $\mathrm{Eu}$ and $\mathrm{Sn}$ atomic radius. However, it has been shown that strain in the ternary $\mathrm{Sn}_{1-x} \mathrm{Eu}_{x} \mathrm{Te}$ can widen the miscibility range up to $\mathrm{x} \sim 16 \%$ [22]. We infer, therefore, that the relatively high interdiffusion values observed here probably result from the strain present in the QDs layer.

Figure 4(b) shows the widths of angular scans and the respective side lengths of each iso-lattice parameter region obtained from $2 \pi / \mathrm{q} / /$ as a function of $(\mathrm{h}, \mathrm{k})$. Although the lateral size of the iso-lattice parameter regions cannot be used to reconstruct the three-dimensional composition profile of QDs - contrary to uncapped 
nanostructures [10,17] - the results of Fig. 4(b) can be employed to draw a twodimensional projection map of in-plane local lattice parameter $\mathrm{a}=\mathrm{a}_{\mathrm{SnTe}} \mathrm{h} / 2$ (considering $|\mathrm{h}|=|\mathrm{k}|)$ - for EuTe islands. In such iso-strain projections the areas with each lattice parameter are drawn following the simple ellipse constraint $\left(\mathrm{x}^{2}+\mathrm{y}^{2} / \mathrm{L}^{2}\right) \leq 1$, where $\mathrm{x}, \mathrm{y}$ and $\mathrm{L}$ are the coordinates and iso-lattice parameter lateral size along the $[1-10]$ and $[2,11]$ directions, here considered the same accordingly to the equal L sizes obtained from measurements in the $\langle 220\rangle$ family of reflections [23]. Figure 4(c) shows the in-plane iso-lattice parameter projections for dots. The projection analysis can be extended by correlating local lattice parameter and composition results from Fig. 4(a). Since in this figure the local composition is obtained for each lattice parameter, this chemical information can be directly correlated to the equivalent lattice parameter projection in Fig. 4(c). The result-a composition projection map-is shown in Fig. 4(d). The resulting projections show that central regions are more Eu-rich than regions at the periphery of dots. Finally, the local in-plane strain $\left(\varepsilon_{/ /}\right)$projection can be obtained by $\varepsilon_{/ /}=\left(\mathrm{a}_{/ /}-\mathrm{a}_{\text {relaxed }}\right) / \mathrm{a}_{\text {relaxed }}$, where $\mathrm{a} / /$ is the local in-plane lattice parameter from projections of Fig. 4(c) and a relaxed is the bulk lattice parameter obtained from Vegard's law for the given EuTe/SnTe concentration of Fig 4(d). Although these maps do not provide the three-dimensional composition profile of EuTe dots, they can be used to infer information about electronic properties of our nanostructures as well as quantum confinement in similar QDs [24].

\subsection{InP islands}

III-V semiconductor alloys show a wide possibility for physical properties tuning as well as different substrate options and various growth techniques. A main issue in III-V islands growth is the interdiffusion of substrate atoms into the islands, which occurs mainly due to the chemical affinity of the elements involved, the high growth temperatures and the elastic energy minimization in the nanostructures.

In this section, we have determined the concentration profile of all chemical species, as well as the strain of InP islands grown on $\mathrm{GaAs}(001)$, by performing resonant X-ray diffraction in grazing incidence geometry. The influence of the growth temperature and indium flux on the profiles was addressed and found to be determinant on the concentration of substrate atoms on the resulting islands, as well as the strain stored on the QDs.

Figure 5 shows AFM images for all InP samples studied in this work. Sample A displays a bimodal size distribution, with few very large (probably incoherent) islands and smaller coherent islands [25]. The formation of large incoherent islands is associated to limited mobility of the adatoms on the (relatively) cold surface of the substrate. Sample B presents a homogeneous distribution of islands covering the entire surface of the sample, while sample $\mathrm{C}$ shows small sparse islands similar to surface roughness [17]. The sample grown with lower In flux - sample D - has shown a lower island density when compared to sample B, and the deposited material is concentrated in slightly larger islands.

Radial scans spanning in reciprocal space in the vicinity of the GaAs (220) inplane reflection for $h=k$ and $l=0$ are shown in Fig. 6 for samples B and D. Such measurements probe the existence of an in-plane lattice parameter gradient inside the islands $[3,10,25]$. The intense $\operatorname{GaAs}(220)$ substrate peak is not shown for clarity. The shoulder at lower $q$ values corresponds to scattering of strained island material that relax from a fully strained condition matching the GaAs lattice parameter $\left(\mathrm{a}_{\mathrm{GaAs}}=5.653 \AA\right)$ towards the $\mathrm{InP}$ unstrained lattice parameter $\left(\mathrm{a}_{\mathrm{InP}}=5.868 \AA\right)$. Scans were performed at the K-edge and below the K-edge, for comparison purposes, for both Ga and As. The variation of scattered intensity observed takes place due 

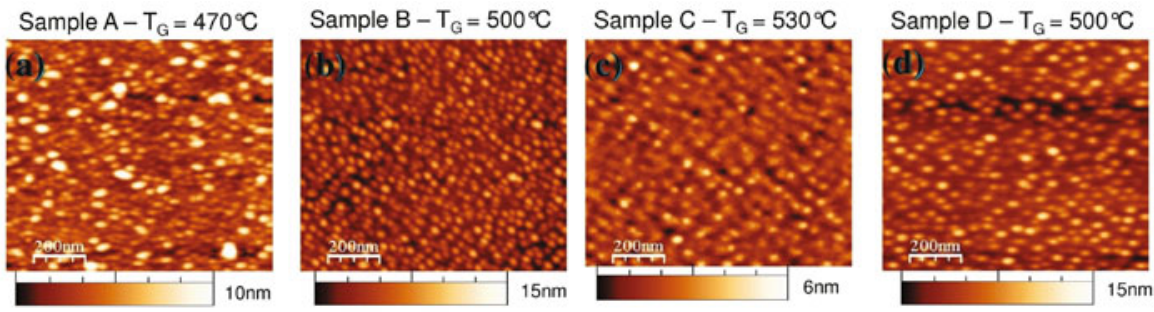

Fig. 5. AFM topographic images of $\mathrm{InP}$ islands obtained in different growth conditions. High In flux was used in samples (a) A- $470{ }^{\circ} \mathrm{C}$, (b) B-500 ${ }^{\circ} \mathrm{C}$, (c) $\mathrm{C}-530^{\circ} \mathrm{C}$ while a low In flux was used in (d) D-500 ${ }^{\circ} \mathrm{C}$.

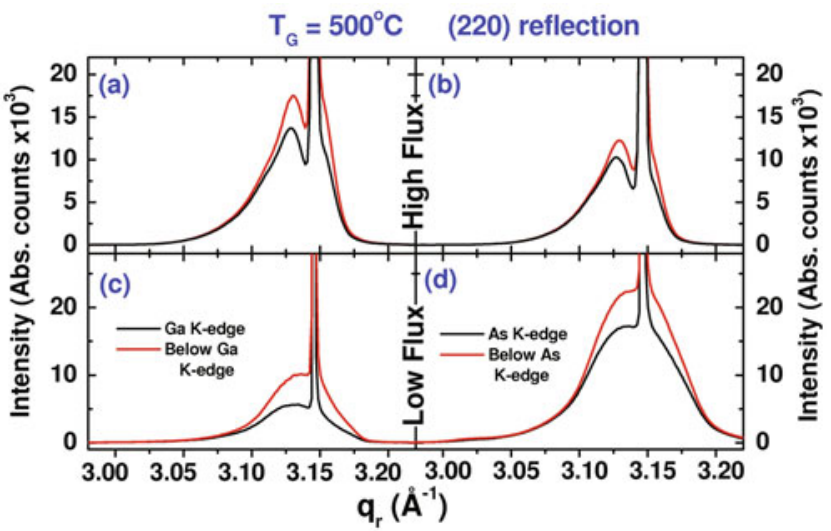

Fig. 6. Radial scans around the (220) reflection shows the variation of intensity in the scans were $\mathrm{Ga}$ or As atoms are present for the samples grown at $500{ }^{\circ} \mathrm{C}$. (a) and (b) show concentration contrast for the high flux sample and (c) and (d) are the results for the low flux sample. Scans on the left were performed below and at the Ga K-edge (a and c) and on the right below and at the As K-edge (b and d).

to changes in the scattering factor for these atoms close to the absorption edges, as shown in Fig. 2(c,d). The intensity observed in Fig. 6 is reduced close to the absorption edges for all graphs, indicating the presence of substrate atoms inside the islands. The contrast is larger for $\mathrm{Ga}$ in both samples, which is expected, since the samples were grown on $\mathrm{P}$ atmosphere, representing an infinite reservoir of this atomic species, whereas the incoming In precursor did not effectively adsorbed on the growth surface for the growth temperatures used, driving the incorporation of Ga into the islands. The matching of the diffraction intensities for all curves were based on radial scans performed in sample A (not shown here), in which the intensity of the peak of incoherent (relaxed) InP islands does not vary.

The energy dependence of the X-ray scattered intensity for the (220) reflection is a function of the structure factor for the zinc blend crystalline structure as well as the concentration of each atomic species and finally, their atomic scattering factors. For GaAs, one obtains the following expression for the scattering structure factor:

$$
I \propto\left|F_{h k l}\right|^{2}=16\left\{\left|f^{(1)}\right|^{2}+\left|f^{(2)}\right|^{2}+f^{(1)} f^{(2)} 2 \cos \left[\frac{\pi}{2}(h+k+l)\right]\right\},
$$

where is the complete atomic scattering factor, $f^{(1)}=f^{0}(q)+f^{\prime}(E)+f^{\prime \prime}(E)$. Depending on the combination of Miller indexes, the reflection is forbidden or not. A 
quick analysis of the possibilities yields the following rules (consider $n$ integer). For all indexes $h, k, l$ being even one obtains two possible types of reflections:

$$
\begin{gathered}
h+k+l=4 n \rightarrow I \propto\left|F_{h k l}\right|^{2}=16\left(f^{(1)}+f^{(2)}\right)^{2}, \\
h+k+l=4 n+2 \rightarrow I \propto\left|F_{h k l}\right|^{2}=16\left(f^{(1)}-f^{(2)}\right)^{2} .
\end{gathered}
$$

The intensity observed at the (220) reflection follows Eq. (5). When quaternary islands are considered the system has 4 (rather than 2) chemical species, that occupy well defined crystallographic sites, i.e., type III(V) atoms replace type III(V) atoms. The diffraction intensity is then given by the square of a coherent sum of the atomic scattering factors from each species weighed by their concentrations:

$$
I=k\left(C_{G a} f_{G a}+C_{A s} f_{A s}+C_{I n} f_{I n}+C_{P} f_{P}\right)^{2},
$$

where indicates the concentration of the n-element and its respective atomic scattering factor and $\mathrm{k}$ is an energy independent constant. Considering that all type III (V) occupy the same position on the crystal, the Ga $(\mathrm{As})$ and $\mathrm{In}(\mathrm{P})$ concentrations are complementary, that is, $C_{G a}+C_{I n}=1\left(C_{A s}+C_{P}=1\right)$. Since neither the In K-edge $(\mathrm{E}=27940 \mathrm{eV})$, or the In L1 edge $(\mathrm{E}=4237 \mathrm{eV})$ and the P K-edge $(0.530 \mathrm{eV})$ are available within the energy range accessible in the beamlines used $(5 \mathrm{keV}-15 \mathrm{keV})$, determination of $\mathrm{Ga}$ and As concentrations as complementary to In and $\mathrm{P}$ is the only way to quantify the concentration of these elements in the islands. As the Xray energy approaches a K-edge of a given element, the atomic scattering factor of this specific chemical species varies considerably, while the other scattering factors for all other elements present in our quaternary system remain nearly constant. For the scans performed at the four selected energies scans $-E 1=10367 \mathrm{eV}$ (Ga edge), $E 2=10268 \mathrm{eV}$ (below Ga edge), $E 3=11867 \mathrm{eV}$ (As edge), $E 4=11751 \mathrm{eV}$ (below As edge) - one can then write:

$$
\begin{aligned}
& \frac{I\left(E_{1}\right)}{I\left(E_{2}\right)}=k 1\left(\frac{C_{G a} f_{G a}\left(E_{1}\right)+C_{A s} f_{A s}+\left(1-C_{G a}\right) f_{I n}+\left(1-C_{A s}\right) f_{P}}{C_{G a} f_{G a}\left(E_{2}\right)+C_{A s} f_{A s}+\left(1-C_{G a}\right) f_{I n}+\left(1-C_{A s}\right) f_{P}}\right)^{2}, \\
& \frac{I\left(E_{3}\right)}{I\left(E_{4}\right)}=k 2\left(\frac{C_{G a} f_{G a}+C_{A s} f_{A s}\left(E_{3}\right)+\left(1-C_{G a}\right) f_{I n}+\left(1-C_{A s}\right) f_{P}}{C_{G a} f_{G a}+C_{A s} f_{A s}\left(E_{4}\right)+\left(1-C_{G a}\right) f_{I n}+\left(1-C_{A s}\right) f_{P}}\right)^{2} .
\end{aligned}
$$

Isolating for $C_{G a}$ and $C_{A s}$, one obtains the concentrations of all four elements, due to the complementary relation between the concentrations of type III (V) atoms:

$$
\begin{gathered}
C_{G a}=B_{G a} \frac{1-\sqrt{\frac{I\left(E_{1}\right)}{I\left(E_{2}\right)}}}{f_{G a}\left(E_{2}\right) \sqrt{\frac{I\left(E_{1}\right)}{I\left(E_{2}\right)}}-f_{G a}\left(E_{1}\right)+f_{I n} \sqrt{\frac{I\left(E_{1}\right)}{I\left(E_{2}\right)}}}, \\
C_{A s}=B_{A s} \frac{1-\sqrt{\frac{I\left(E_{3}\right)}{I\left(E_{4}\right)}}}{f_{A s}\left(E_{4}\right) \sqrt{\frac{I\left(E_{3}\right)}{I\left(E_{4}\right)}}-f_{A s}\left(E_{3}\right)+f_{P} \sqrt{\frac{I\left(E_{3}\right)}{I\left(E_{4}\right)}}} .
\end{gathered}
$$

The constants $B_{G a}$ and $B_{A s}$ contain all the variables that do not change as the photon energy is tuned to the element edge. Evaluating the concentrations of Ga and As and, consequently, In and P, one obtains the profiles shown in Fig. 7.

In samples $\mathrm{A}$ and $\mathrm{B}$ the $\mathrm{Ga}$ and As concentration falls rapidly with lattice parameter, indicating the presence of substrate atoms only on the base of the islands, 


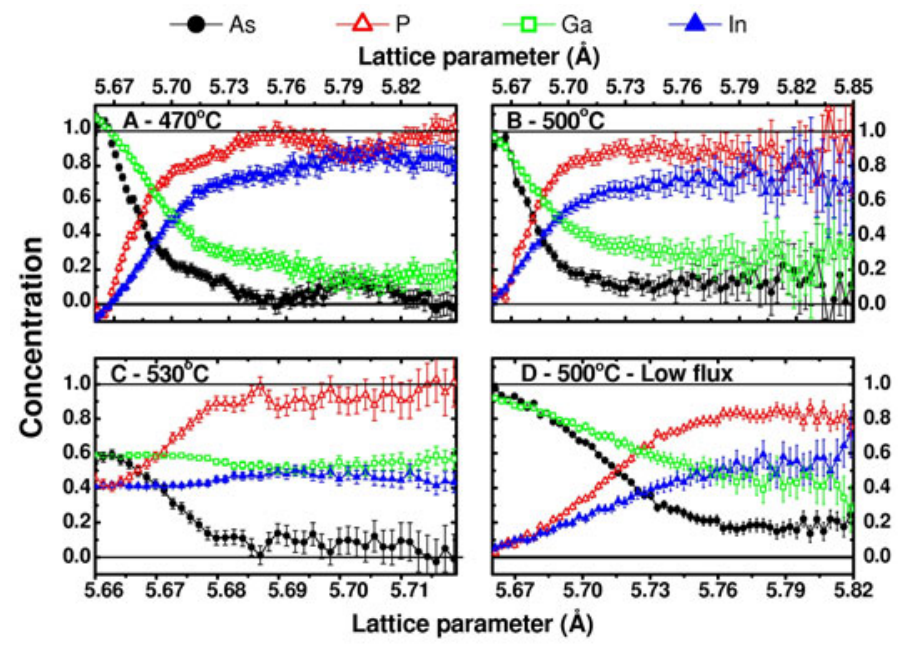

Fig. 7. Element concentration as function of lattice parameter for the four elements present in all samples.

where the lattice parameter is closer to that of the GaAs substrate. The high flux sample grown at $530^{\circ} \mathrm{C}$ (sample C) presents a high Ga concentration throughout the whole island, forming an InGaP ternary alloy. In sample $\mathrm{D}$, the growth of islands at $500{ }^{\circ} \mathrm{C}$ with low In flux also leads to higher Ga content inside the islands in comparison to sample A. Such behavior indicates the presence of a surface kinetic diffusion mechanism that takes place during island growth and allows Ga atoms to replace missing In atoms in the structure due to the reduced income of the latter species. An interesting comparison can be drawn between samples grown at $500{ }^{\circ} \mathrm{C}$ with different In-fluxes. The Ga concentration in sample D remains high up to larger lattice parameters than sample B, indicating considerable migration of substrate atoms to the islands to compensate the small inflow of In atoms. The higher As concentration help to decrease the overall strain, and therefore a higher concentration of As atoms are incorporated into the island, forming an As-Ga rich alloy with less strain.

The concentration profiles for the four samples reveals the dynamics of the In precursor as it reaches the growth front. For samples A (high flux, $470{ }^{\circ} \mathrm{C}$ ) and $\mathrm{B}$ (high flux, $500^{\circ} \mathrm{C}$ ) the In precursor is cracked once it reaches the surface, producing In adatoms that remain attached to it. The main difference between these samples is not compositional but structural, since reduced adatom mobility is observed for sample A, leading to the formation of incoherent large islands. Sample C (high In flux, $530^{\circ} \mathrm{C}$ ) is grown at a temperature in which In precursor leaves the surface before complete cracking occurs, with considerable In desorption. This reduced availability of type III atoms causes large migration of Ga substrate atoms to the islands, resulting in the large concentration of $\mathrm{Ga}$ in the whole island, forming a ternary alloy (InGaP) rather than pure InP islands.

At specific $\mathrm{qr}_{\mathrm{r}}$ positions, meaning specific local lattice parameter $\left(a^{\prime}\right)$ values, angular scans were performed to assess the lateral size of the QDs $[3,25]$. The island lateral size $L$ is inversely proportional to the width at half maximum $\sigma$ of the angular scan, so that $L=2 \pi / \sigma$. Figure 8(a) shows angular scans for sample B at indicated $a^{\prime}$ values. The islands are wider at the base, as seen by AFM profiles, presenting an angular scan with smaller $\sigma$. As $a^{\prime}$ relaxes to $a_{\mathrm{InP}}(5.86 \AA)$, increases, a clear indication of a narrower scattering region, that is, the top of the islands. Figures 8(b) and 8(c) show the dependence of $L$ with $a^{\prime}$ for samples B and C, respectively. QDs present in 

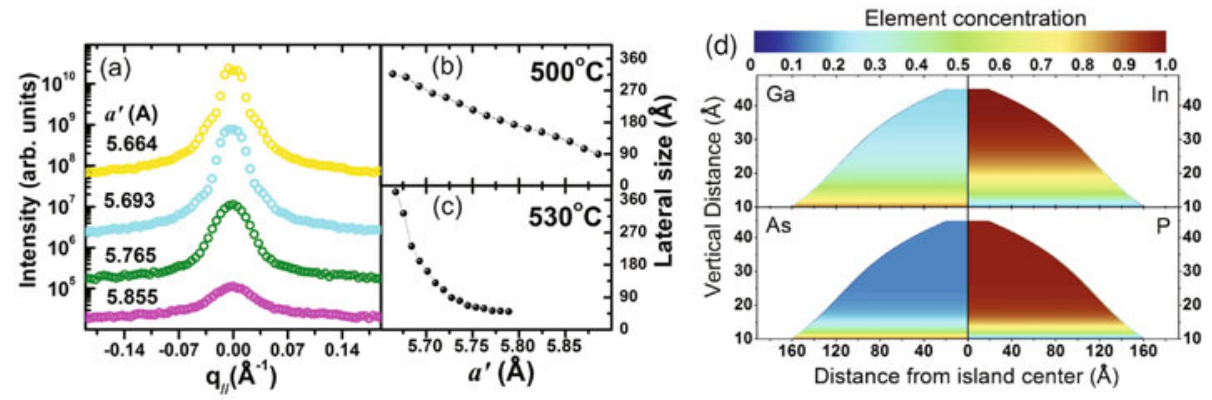

Fig. 8. (a) Angular scan profiles obtained for sample B at distinct in-plane lattice parameter (a') conditions. Lateral size extracted from angular scan widths for samples B and C are shown in (b) and (c), respectively. (d) Elemental concentration map in real space retrieved by the combination of X-ray diffraction results and AFM average profiles for the islands of sample B $[10,17]$.

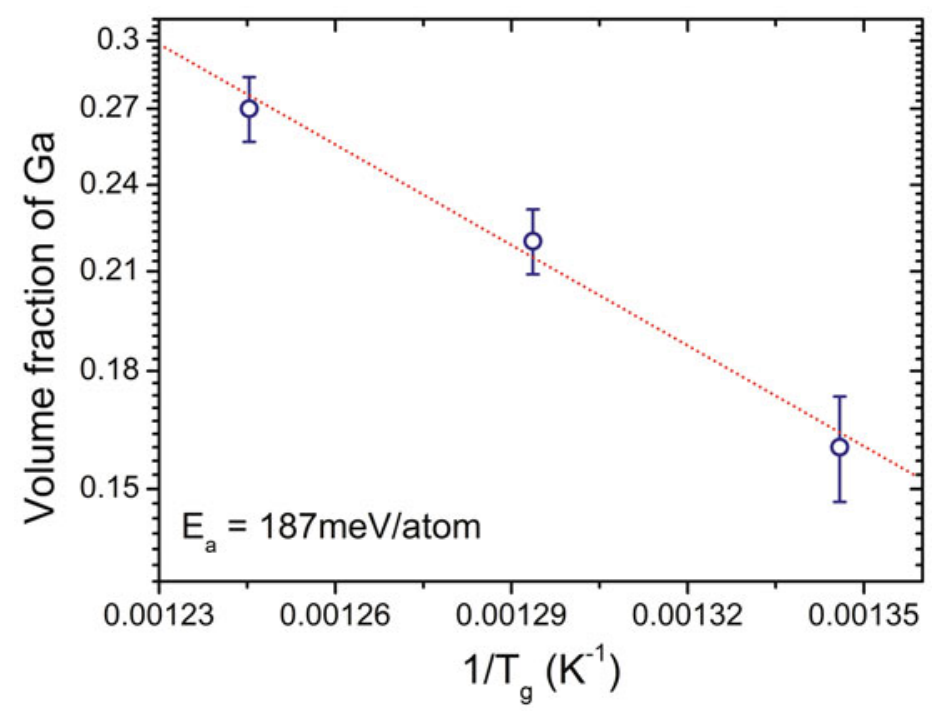

Fig. 9. Arrhenius plot from the Ga volume fraction integrated for islands in samples A, B, $\mathrm{C}$, allowing to infer the activation energy for incorporation of $\mathrm{Ga}$ atoms into the islands.

sample B show a monotonic decrease of $L$ as $a^{\prime}$ increases, indicating that the lattice relaxes elastically as it grows away from the substrate. As for C, a more pronounced descent occurs, indicating that a second mechanism of lattice relaxation is present. Comparing the slope of $L$ as a function of $a^{\prime}$ for samples $\mathrm{B}$ and $\mathrm{C}$ we conclude that relaxation is mainly elastically driven for the former and chemically driven for the latter [17].

In order to obtain average numbers of chemical concentration and strain relaxation, a plot of chemical composition as a function of height and width is necessary. From the AFM profile, the height $\mathrm{h}$ of a typical island was related to its lateral width $L$, corrected to yield maximum $L$ at $\mathrm{h}=0$. Angular scans allow for correlating the width $L$ to the local lattice parameter $a$ '. The chemical concentration on the islands obtained from the radial scans in Fig. 6 was also displayed as a function of $a$ ' [Fig. 7]. The data of these three analyses were combined to build maps where the chemical concentration averaged over $L$ for each iso-lattice parameter region in the 
islands is shown as function of height [Fig. 8(d)]. When combining the analysis from the AFM with the one from the angular scans, an error of $10 \%$ in the island dimensions is expected due to convolution of the islands with the tip (influences the AFM profile) and to an amorphous oxide cap on the QDs, which results in a maximum width from AFM larger than the one obtained from the angular scans, since the amorphous oxide layer does not contribute to . Lateral concentration profiles [26] were not probed in the direction of the radial scan, so the results presented provide only the vertical variation of concentration, averaged over the width $L$ of the QDs.

Finally, the Ga concentration can be evaluated for islands in each of the samples grown at high In flux. By plotting the volume fraction of Ga inside samples A, B and C as a function of the inverse of the growth temperature (Arrhenius plot) the activation energy $\left(\mathrm{E}_{\mathrm{a}}\right)$ for incorporation of one Ga atom inside the InP islands can be extracted for the CBE growth conditions used. The obtained $\mathrm{E}_{\mathrm{a}}$ has a value of $187 \mathrm{meV} /$ atom, very reduced with respect to values of $\mathrm{Ga}$ incorporation via bulk diffusion, typically of a few eV $[27,28]$. Such result points out for an important contribution from surface kinetics to the incorporation of Ga inside the islands, in agreement with the In desorption scenario expected for temperatures around $500^{\circ} \mathrm{C}$.

\section{Conclusions}

Two systems were studied in this work, representing variations of the possibilities of combined anomalous X-ray scattering and grazing incidence diffraction analysis of epitaxial nanostructures. The first system, EuTe:SnTe, was studied in its chemical aspects by directly accessing the vicinity of the $\mathrm{Eu} \mathrm{L}_{3}$ edge. It was shown that coherent SnTe capped EuTe islands present a considerable degree of interdiffusion of Sn, which can affect the magnetic and optoeletronic behavior of such nanostructures. Although a three dimensional concentration and strain map could not be directly retrieved, projection maps were drawn, allowing the visualization of the lateral sizes of isolattice parameter and iso-composition regions. Such information can already provide clues for future analysis of confined electronic states in similar QD systems.

The second system, in which a quaternary alloy is formed by deposition of InP on GaAs, was studied by means of complementary anomalous X-ray diffraction using the $\mathrm{Ga}$ and $\mathrm{As} \mathrm{K}$-edges. The interdiffusion of substrate atoms into the islands grown at different temperatures and growth rates was discussed, allowing to understand the possibilities of fine tuning of the optoelectronic properties in these QDs. Finally, the activation energy for the incorporation of Ga adatoms in the high flux deposited islands was extracted, showing that Ga migration into these islands has a relevant surface diffusion component.

This work was supported by ABTLuS (Brazilian Synchrotron Association), FAPESP, and $\mathrm{CNPq}$ (Brazilian fuding agencies).

\section{References}

1. Y. Waseda, Anomalous X-ray Scattering for Materials Characterization (Springer, 2002)

2. J. Als-Nielsen, D. McMorrow, Elements of Modern X-ray Physics, 2nd edn. (Wiley, 2001)

3. I. Kegel, T.H. Metzger, P. Fratz, J. Peisl, A. Lorke, J.M. Garcia, P.M. Petroff, Europhys. Lett. 45, 222 (1999)

4. I. Robinson, R. Harder, Nature Materials 8, 291 (2009)

5. M.S. Leite, A. Malachias, S.W. Kycia, T.I. Kamins, R.S. Williams, G. Medeiros-Ribeiro, Phys. Rev. Lett. 100, 226101 (2008) 
6. V.I. Klimov, A.A. Mikhailovsky, Su Xu, A. Malko, J.A. Hollingsworth, C.A. Leatherdale, H.-J. Eisler, M.G. Bawendi, Science 290, 314 (2000)

7. A. Badolato, K. Hennessy, M. Atatüre, J. Dreiser, E. Hu, P.M. Petroff, A. Imamoglu, Science 308, 1158 (2005)

8. A.G. Cullis, D.J. Norris, T. Walther, M.A. Migliorato, and M. Hopkinson, Phys. Rev. B 66, 081305 (2002)

9. G.W. Bryant, M. Zieliñski, N. Malkova, J. Sims, W. Jaskólski, J. Aizpurua, Phys. Rev. Lett. 105, 067404 (2010)

10. R. Magalhães-Paniago, G. Medeiros-Ribeiro, A. Malachias, S. Kycia, T.I. Kamins, R.S. Williams, Phys. Rev. B 66, 245312 (2002)

11. A. Malachias, W.N. Rodrigues, M.V.B. Moreira, S. Kycia, R. Magalhaes-Paniago, J. Phys. D 36, 249 (2003)

12. T.U. Schülli, J. Stangl, Z. Zhong, R.T. Lechner, M. Sztucki, T. H. Metzger, G. Bauer, Phys. Rev. Lett. 90, 066105 (2003)

13. T.U. Schülli, M. Stoffel, A. Hesse, J. Stangl, R.T. Lechner, E. Wintersberger, M. Sztucki, T.H. Metzger, O.G. Schmidt, G. Bauer, Phys. Rev. B 71, 035326 (2005)

14. A. Malachias, M. Stoffel, M. Schmidbauer, T.Ü. Schulli, G. Medeiros-Ribeiro, O.G. Schmidt, Rogerio Magalhães-Paniago, T.H. Metzger, Phys. Rev. B 82, 035307 (2010)

15. M. Floyd, Y. Zhang, K.P. Driver, J. Drucker, P.A. Crozier, D.J. Smith, Appl. Phys. Lett. 82, 1473 (2003)

16. B. Diaz, A. Malachias, P.H.O. Rappl, E. Abramof, V.A. Chitta, A.B. Henriques, J. Cryst. Growth 312, 2828 (2010)

17. L.N. Coelho, R. Magalhães-Paniago, A. Malachias, J.G. Zelcovit, M.A. Cotta, Appl. Phys. Lett. 92, 021903 (2008)

18. W. Heiss, G. Prechtl, G. Springholz, Phys. Rev. B 63, 165323 (2001)

19. L.K. Hanamoto, et al., J. Phys. Cond. Mat. 16, 5597 (2004)

20. H. Kepa, Phys. Rev. B 68, 024419 (2003)

21. G. Springholz, et al., Science 282, 734 (1998)

22. E. Abramof, P.H.O. Rappl, A.Y. Ueta, Physica E 20, 462 (2004)

23. L. Villegas-Lelovsky, M.D. Teodoro, V. Lopez-Richard, C. Calseverino, A. Malachias, E. Marega, B.L. Liang, Yu I. Mazur, G.E. Marques, C. Trallero-Giner, G.J. Salamo, Nanoscale Res. Lett. 6, 56 (2011)

24. M.D. Teodoro, A. Malachias, G.E. Marques, V. Lopez-Richard, E. Marega Jr., Yu. I. Mazur, M. Benamara, G.J. Salamo (submitted)

25. A. Malachias, R. Magalhães-Paniago, B.R. A. Neves, W.N. Rodrigues, M.V.B. Moreira, H.-D. Pfannes, A.G. de Oliveira, S. Kycia, T.H. Metzger, Appl. Phys. Lett. 79, 4342 (2001)

26. A. Malachias, S. Kycia, G. Medeiros-Ribeiro, R. Magalhães-Paniago, T.I. Kamins, R.S. Williams, Phys. Rev. Lett. 91, 176101 (2003)

27. J.F. Wager, J. Appl. Phys. 69, 3022 (1991)

28. H. Yang, V.P. LaBella, D.W. Bullock, Z. Ding, J.B. Smathers, P.M. Thibado, J. Cryst. Growth 201, 88 (1999) 